\title{
Achievement of essentially full spatial coherence in a high-average-power soft-x-ray laser
}

\author{
Y. Liu,${ }^{1,4}$ M. Seminario, ${ }^{2}$ F. G. Tomasel, ${ }^{2}$ C. Chang, ${ }^{3,4}$ J. J. Rocca, ${ }^{2}$ and D. T. Attwood, ${ }^{1,3,4}$ \\ ${ }^{1}$ Applied Science and Technology Graduate Program, University of California, Berkeley, California 94720 \\ ${ }^{2}$ Department of Electrical and Computer Engineering, Colorado State University, Fort Collins, Colorado 80523 \\ ${ }^{3}$ Department of Electrical Engineering and Computer Science, University of California, Berkeley, California 94720 \\ ${ }^{4}$ Center for X-ray Optics, Lawrence Berkeley National Laboratory, Berkeley, California 94720
}

(Received 21 July 2000; published 5 February 2001)

\begin{abstract}
We report an observation of essentially full spatial coherence in a high average power soft-x-ray laser. Rapid coherence buildup due to strong refractive antiguiding in a long plasma column is experimentally demonstrated. This allows the generation of fully coherent, milliwatt-level average power soft-x-ray radiation by a tabletop device. The peak brightness of this laser reaches $2 \times 10^{25}$ photons s $\mathrm{mm}^{-2} \mathrm{mrad}^{-2}$ within $0.01 \%$ spectral bandwidth, making it one of the brightest soft-x-ray sources available.
\end{abstract}

DOI: 10.1103/PhysRevA.63.033802

PACS number(s): 42.55.Vc

Applications such as high-resolution microscopy, interferometry, lithography, and holography motivate the development of advanced light sources at soft-x-ray wavelengths. Current approaches for the generation of high brightness coherent radiation in this spectral region include undulators at modern synchrotron radiation (SR) facilities [1], high-order harmonic generation $(\mathrm{HHG})$ of optical lasers $[3,4]$, and soft$\mathrm{x}$-ray lasers [2]. The degree of spatial coherence of radiation plays a critical role in many of the most important applications. For SR sources, high spatial coherence is achieved at the expense of photon flux by spatial filtering [1]. HHG sources driven by coherent optical lasers have shown high spatial coherence with average powers reaching the order of microwatts by using a phase-matching technique [4]. Compared with SR and HHG sources, soft-x-ray lasers have substantially higher pulse energy and narrower linewidth. However, to date they have been characterized by rather low spatial coherence [5-9]. The demonstration of nearly full spatial coherence has been one of the main goals of soft- $x-$ ray laser research.

Soft-x-ray laser beams are generally limited to single-pass or double-pass amplification of spontaneous emission (ASE) through the plasma. From the van Cittert-Zernike theorem [10], a high degree of spatial coherence from an ASE-based laser can be achieved when the gain medium has a Fresnel number less than unity. However, this is difficult to achieve in a plasma column, unless some forms of spatial filtering are used $[11,12]$. Here we experimentally demonstrate that refraction in a plasma with sharp density gradients can reduce the effective transverse source size significantly and result in essentially full spatial coherence. Although theoretical results have suggested that refractive antiguiding and gain guiding along a long plasma column could result in improved spatial coherence [13-15], in previous experiments the coherence buildup was limited to values significantly below full coherence $[7,16]$. In the present work, we utilized fast capillary discharge excitation to produce plasma columns with both very high axial uniformity and the length-todiameter ratio exceeding 1000:1, in which strong refractive antiguiding makes it possible to achieve essentially full spatial coherence with a plasma column length of $36 \mathrm{~cm}$.
The laser beam in our experiments is generated by excitation of an Ar-filled capillary channel with a fast discharge current pulse that rapidly compresses the plasma to form a dense and hot column with a large density of Ne-like ions $[17,18]$. Collisional electron impact excitation of the Ne-like ions produces a population inversion between the $3 p\left({ }^{1} S_{0}\right)$ and $3 s\left({ }^{1} P_{1}^{0}\right)$ levels, resulting in amplification at $46.9 \mathrm{~nm}$. The experiments are conducted utilizing aluminum oxide capillary channels $3.2 \mathrm{~mm}$ in diameter and up to $36 \mathrm{~cm}$ in length, filled with preionized Ar gas at a pressure of $\sim 59 \mathrm{~Pa}$. The plasma columns are excited by current pulses of $\sim 25$ kA peak amplitude, with a $10-90 \%$ rise time of approximately $40 \mathrm{~ns}$. The setup is similar to that used in previous experiments $[19,20]$. The excitation current pulse was produced by discharging a water dielectric capacitor through a spark gap switch connected in series with the capillary load. The laser is very compact and occupies a table area of only $0.4 \times 1 \mathrm{~m}^{2}$. Efficient extraction of energy is obtained by operating the laser in a highly saturated regime. The laser pulse energy increases nearly exponentially as a function of plasma column length, until the beam intensity reaches the gain saturation intensity at a plasma column length of about $14 \mathrm{~cm}$ [18]. For longer plasma columns, the laser average pulse energy increases linearly with length from $0.075 \mathrm{~mJ}$ for a plasma column $16 \mathrm{~cm}$ in length to $0.88 \mathrm{~mJ} \quad(>2$ $\times 10^{14}$ photons/pulse) for a plasma column length of $34.5 \mathrm{~cm}$ [20]. Average laser powers of $3.5 \mathrm{~mW}$ are obtained when operating the laser at a repetition rate of $4 \mathrm{~Hz}$.

The spatial coherence of a quasimonochromatic light

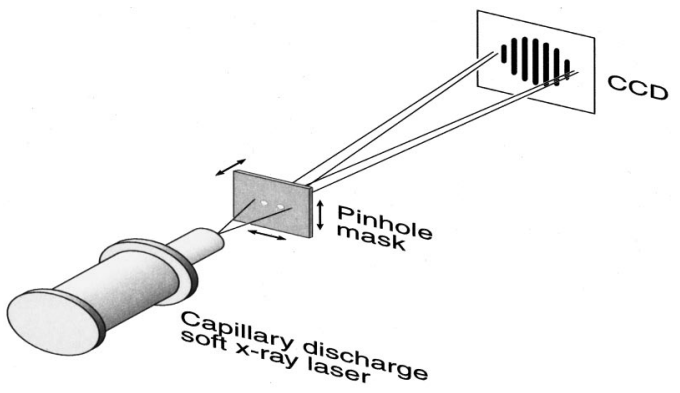

FIG. 1. Schematic representation of the experimental setup used in the two-pinhole interference coherence measurements. 

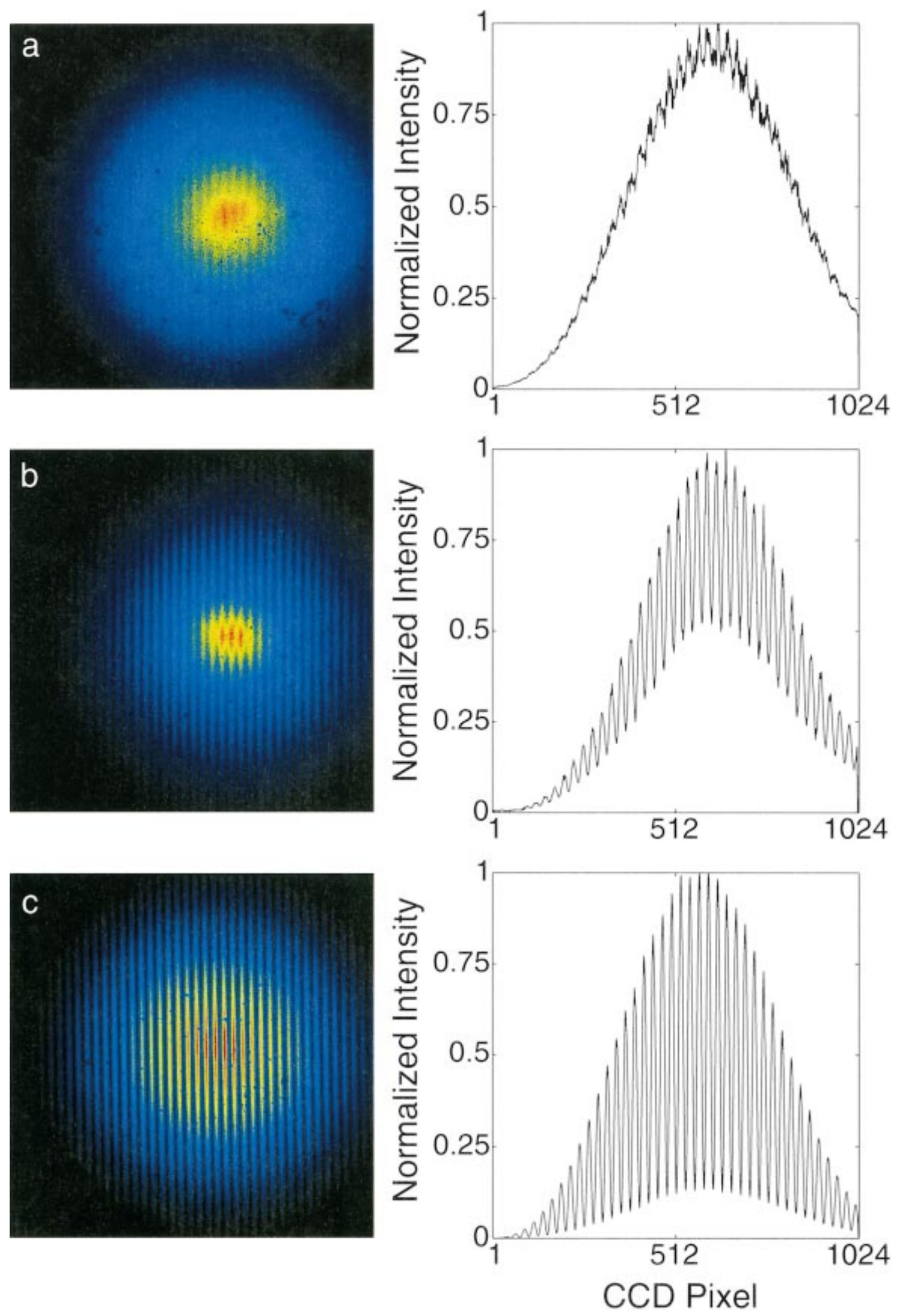

FIG. 2. (Color) Interferograms and their lineouts showing the coherence buildup of the laser beam with increasing capillary length. The capillary lengths are (a) 18, (b) 27 , and (c) $36 \mathrm{~cm}$. The lineouts are obtained by vertically integrating 15 pixels of the CCD images. 

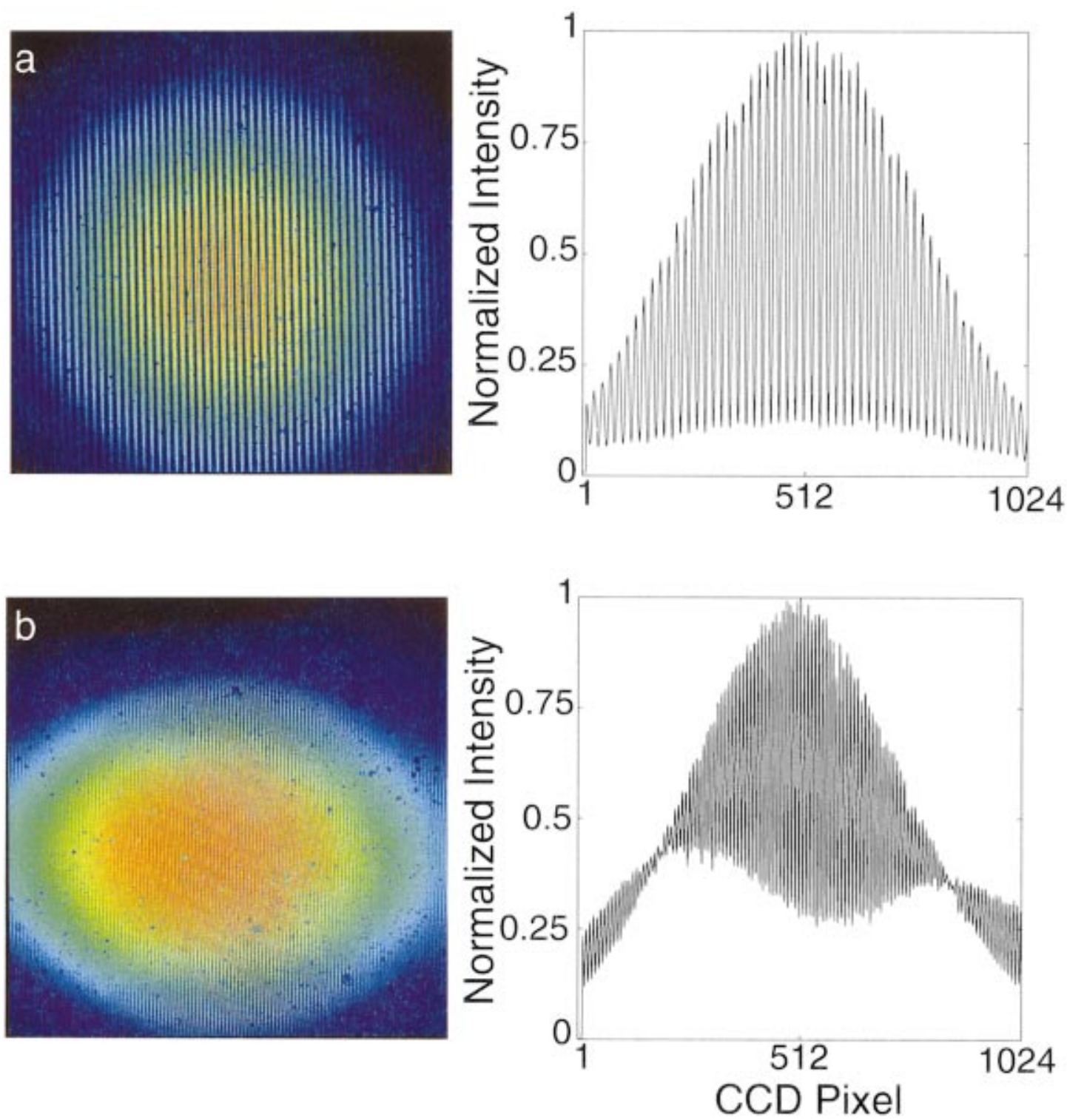

FIG. 3. (Color) Interferograms and their lineouts obtained with two pinholes located at $15.7 \mathrm{~cm}$ from the capillary exit. The pinhole separations are (a) 300 and (b) $680 \mu \mathrm{m}$.

source is characterized by the cross correlation of fields across the output wavefront, which can be described in terms of the normalized complex degree of coherence $\mu_{12}$ [10]. In a two-pinhole interference experiment, the fringe visibility, defined as $V=\left(I_{\max }-I_{\min }\right) /\left(I_{\max }+I_{\min }\right)$, where $I_{\max }$ and $I_{\min }$ are the maximum and minimum intensities of the fringe pattern, as a function of pinhole separation is proportional to the modulus of $\mu_{12}$ [21]. This was demonstrated in experiments by Thompson and Wolf with partially coherent visible light [22]. Similar measurements were conducted recently in SR sources [23]. A variation of this method, a two-slit experiment, had been used in measuring the spatial coherence of both X-ray lasers $[8,24]$ and HHG sources [25]. The laser used in our experiment has sufficiently high pulse energy for us to perform a two-pinhole interference experiment with a single laser pulse.

The setup used in our two-pinhole interference experiment is shown in Fig. 1. The pinhole masks consisted of pairs of $10-\mu \mathrm{m}$-diam pinholes laser-drilled at selected separations on 12.5- $\mu \mathrm{m}$-thick stainless-steel substrates (National Aperture Inc., NH). Measurements were conducted placing the masks at distances of 15 and $40 \mathrm{~cm}$ from the exit of the capillary. An $x-y$ translation stage was used to position the pinholes with respect to the beam. The interference patterns were recorded with a thermoelectrically cooled, back-thinned charge-coupled device $(\mathrm{CCD})$ having a $1024 \times 1024$ pixel array (SI-003A, Scientific Imaging Technologies, Tigal, OR). The distance from the pinhole plane to the CCD was $300 \mathrm{~cm}$. This distance was selected to assure that the CCD's spatial resolution $(25-\mu \mathrm{m}$ pixel size) is sufficient to resolve the finest interference fringes, while recording essential features of the pinhole diffraction patterns.

The interference patterns recorded by the CCD contain an underlying background that is due to spontaneously emitted radiation from the hot plasma. To reduce its effect, we recorded the background after acquiring each interferogram. 


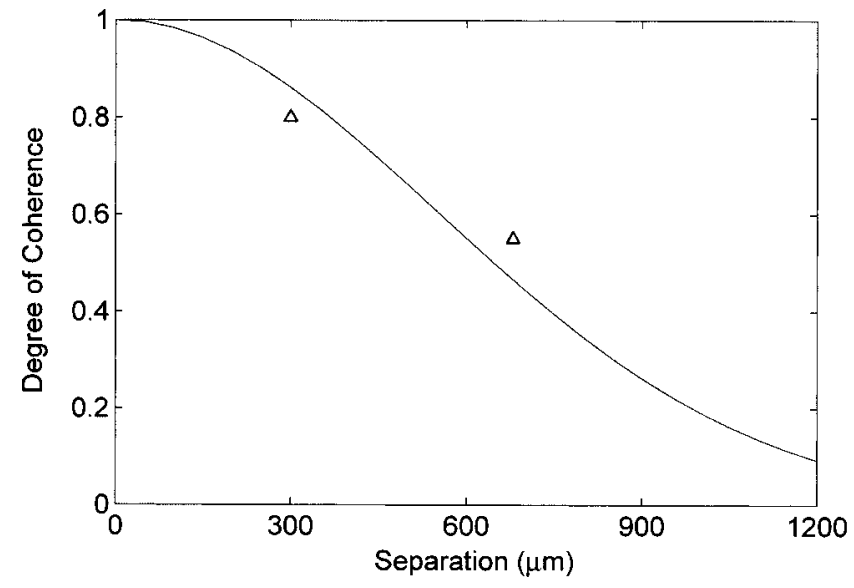

FIG. 4. Measured degree of coherence, $\left|\mu_{12}(\Delta x)\right|$, of the laser beam vs separation between two pinholes at $15.7 \mathrm{~cm}$ from the exit of the capillary. The solid line is for a Gaussian profile $\left|\mu_{12}(\Delta x)\right|$ with a coherence radius $R_{c}=550 \mu \mathrm{m}$.

This was done by increasing the gas discharge pressure to $\sim 130 \mathrm{~Pa}$, which quenches the laser line while maintaining the background emission. Final interferograms were obtained by subtracting the recorded backgrounds from the original interferograms. This procedure also removes thermal "dark counts" of the CCD. The background is, however, somewhat weaker in the higher pressure shots, thus the background removal is not complete. Therefore, the fringe visibility is always somewhat undervalued. As a result, the highest spatial coherence values reported herein, which are sensitive to a small amount of background, constitute a conservative evaluation of the spatial coherence of the source.

Comparative interferograms corresponding to increasing capillary lengths of 18, 27, and $36 \mathrm{~cm}$ are shown in Fig. 2, with their corresponding lineouts. A mask with a pinhole separation of $200 \mu \mathrm{m}$ was used in all three measurements. The mask was positioned at a distance of $40 \mathrm{~cm}$ from the capillary exit. The interferograms consist of two almost entirely overlapped Airy patterns, modulated by the interference between them. The expected coherence buildup with increasing capillary length is clearly observed. The fringe visibility increases from 0.05 for the $18-\mathrm{cm}$-long capillary to 0.33 for the $27-\mathrm{cm}-$ long plasma, and reaches 0.8 for the $36-\mathrm{cm}$ capillary. Assuming a Gaussian profile $\left|\mu_{12}\right|$, the coherence radii [26] for the three capillary lengths are 80, 135, and $300 \mu \mathrm{m}$, respectively. Although the last number is likely to be significantly underestimated as a result of the background error, it is quite clear that the coherence radius scales much faster than linearly with capillary length. This is evidence of refractive mode selection as gain guiding alone provides only a linearly increasing coherence radius $[13,15]$.

Radiation traveling along a plasma tends to be refracted out of the central region, where the amplification process takes place. With the presence of strong refraction, only radiation that propagates near the axis experiences substantial gain and contributes to the output of the laser. Therefore, at the expense of the effective gain, refraction provides a modeselection mechanism that improves the spatial coherence of soft-X-ray lasing. With the assistance of refraction, a coher- ence radius comparable to the beam size was achieved with 36-cm-long capillary length. Evidence of near full spatial coherence requires measurements using pinholes with separation comparable to the beam size. To clarify the point, we positioned the pinholes closer $(15.7 \mathrm{~cm})$ to the capillary exit. The spatial profile of the laser beam at this position was previously measured [20] and verified during these experiments by scanning a single pinhole across the beam. Refraction causes a ring-shaped beam profile with a peak-to-peak diameter of approximately $950 \mu \mathrm{m}$. Figures 3(a) and 3(b) show the obtained interferograms and their lineouts with pinhole separations of 300 and $680 \mu \mathrm{m}$. In Fig. 3(a), visibility as high as 0.8 is observed. In Fig. 3(b), the large pinhole separation, combined with laser divergence, causes a large displacement of the two Airy patterns. The large visibility variations in different regions of the interferogram are the result of the intensity differences between the partially overlapped Airy patterns [21]. Maximum fringe visibility, $\sim 0.55$, occurs where the intensities of the two Airy patterns are equal. Zero visibility occurs where there is a null in one of the Airy patterns. $\left|\mu_{12}\right|$, determined from the maximum value of the visibility, is equal to 0.8 and 0.55 , respectively.

These results indicate a very high degree of spatial coherence throughout practically the entire laser beam. Figure 4 shows the experimental data together with a Gaussian profile $\left|\mu_{12}\right|$ curve with a coherence radius $R_{c}=550 \mu \mathrm{m}$. Considering the small size of the laser beam, we have observed a spatial coherent area containing almost half of the entire laser power, corresponding to a coherent power of more than 1 $\mathrm{mW}$. This extraordinarily high degree of spatial coherence, combined with the high average power and narrow linewidth, makes this laser a powerful coherent photon source for applications. A stricter convention, sometimes used to define coherent area, allows $\left|\mu_{12}\right|$ to drop only to a value of $0.88\left(\sim e^{-1 / 8}\right)[10]$. Use of this stricter criteria would reduce the coherence radius to $\sim R_{c} / 2$. Even so, about $\frac{1}{8}$ of the total power, or $\sim 0.4 \mathrm{~mW}$, is spatially coherent. Moreover, since this high coherent power is generated in only four pulses per second, with a pulsewidth of 1.5 ns each, the laser's peak coherent power is estimated to reach $6 \times 10^{4} \mathrm{~W}$. Assuming focusing with $f / 10$ optics, this peak coherent power would produce a diffraction-limited focal spot with intensity reaching $2 \times 10^{13} \mathrm{~W} \mathrm{~cm}^{-2}$. A coherent source should also be temporally coherent. This laser has a spectral bandwidth of $\Delta \lambda / \lambda \leqslant 1 \times 10^{-4}$, corresponding to a longitudinal coherence length longer than $300 \mu \mathrm{m}$, sufficient for most applications. Therefore, it can be regarded as temporally coherent.

An additional parameter useful for characterizing a light source, and closely related to spatial coherence, is spectral brightness. Previous experiments on beam divergence have shown that this laser can be well approximated as originated from a virtual source located $\sim 5 \mathrm{~cm}$ inside the capillary. We can estimate the size of this source using the van CittertZernike theorem. To produce the same Gaussian coherence profile with $R_{c}=550 \mu \mathrm{m}$, the source should have a diameter (root mean square) of $d_{s}=\lambda z / \pi R_{c}=5.4 \mu \mathrm{m}$. With a measured divergence angle of $7 \operatorname{mrad}(2 \theta)$ [27], the brightness of this source is then $\sim 1.6 \times 10^{17}$, in units of photons $\mathrm{s}^{-1} \mathrm{~mm}^{-2} \mathrm{mrad}^{-2}$ within $0.01 \%$ spectral bandwidth. 
The peak brightness of this laser reaches a value of 2 $\times 10^{25}$, making it one of the brightest soft-x-ray sources, all the more remarkable in that it is entirely contained on a single optical bench. No other soft-x-ray source, independent of its size, is presently capable of simultaneously generating such high average coherent power and peak spectral brightness.

In summary, we have observed an extraordinarily high degree of spatial coherence in high average power soft-x-ray laser beams produced by a tabletop device. The results were obtained by single-pass laser amplification in a very long capillary plasma column using intrinsic mode-selection mechanisms. The availability of full spatial coherence in tabletop soft-x-ray laser beams with high average power and extremely high spectral brightness opens new opportunities in science and technology.

We gratefully acknowledge the support from the National Science Foundation, the Department of Energy, and Air Force Office of Scientific Research.
[1] D. T. Attwood, Soft X-rays and Extreme Ultraviolet Radiation (Cambridge University Press, New York, 1999).

[2] J. J. Rocca, Rev. Sci. Instrum. 70, 3799 (1999).

[3] Ch. Spielmann et al., Science 278, 661 (1997).

[4] A. Rundquist et al., Science 280, 1412 (1998).

[5] J. E. Trebes et al., Phys. Rev. Lett. 68, 588 (1992).

[6] Y. Kato et al., in Ultrashort Wavelength Lasers, edited by S. Suckewer (SPIE, Bellingham, 1992), pp. 56-64.

[7] M. C. Marconi et al., Phys. Rev. Lett. 79, 2799 (1997).

[8] R. E. Burge et al., J. Opt. Soc. Am. B 14, 2742 (1997); 15, 1620 (1998); 15, 2515 (1998).

[9] More papers can be found in X-Ray Lasers 1996, edited by S. Svanberg and C-G. Wahlström (Institute of Physics, Bristol, 1996); X-Ray Lasers 1998, edited by Y. Kato, H. Takuma, and H. Daido (Institute of Physics, Bristol, 1998).

[10] M. Born and E. Wolf, Principles of Optics, 7th ed. (Cambridge University Press, Cambridge, 1999).

[11] D. T. Attwood, in Proceedings of the First Symposium on the Applications of Laboratory X-Ray Lasers, edited by N. M. Ceglio (Lawrence Livermore National Laboratory, Livermore, CA, 1985), CONF-850293-Abstracts, pp. 74-83.

[12] M. D. Rosen, J. E. Trebes, and D. L. Matthews, Comments Plasma Phys. Control. Fusion 10, 245 (1987).

[13] R. A. London, Phys. Fluids 31, 184 (1988).
[14] R. A. London, M. Strauss, and M. D. Rosen, Phys. Rev. Lett. 65, 563 (1990).

[15] P. D. Gasparyan, F. A. Starikov, and A. N. Starostin, Usp. Fiz. Nauk 168, 843 (1998) [Phys. Usp. 41, 761 (1998)].

[16] P. Amendt, M. Strauss, and R. A. London, Phys. Rev. A 53, R23 (1996).

[17] J. J. Rocca et al., Phys. Rev. Lett. 73, 2192 (1994).

[18] J. J. Rocca et al., Phys. Rev. Lett. 77, 1476 (1996).

[19] B. R. Benware et al., Phys. Rev. Lett. 81, 5804 (1998).

[20] C. D. Macchietto, B. R. Benware, and J. J. Rocca, Opt. Lett. 24, 1115 (1999).

[21] J. W. Goodman, Statistical Optics (Wiley, New York, 1985), pp. $171-187$.

[22] B. J. Thompson and E. Wolf, J. Opt. Soc. Am. 47, 895 (1957).

[23] C. Chang et al., Opt. Commun. 182, 25 (2000).

[24] P. Lu et al., Phys. Rev. A 58, 628 (1998).

[25] T. Ditmire et al., Phys. Rev. Lett. 77, 4756 (1996).

[26] In this paper, we use coherence radius $R_{c}$ to characterize the transverse coherence length. The definition of $R_{c}$ is following the convention of coherence area $A_{c}$ used in Ref. [21] as $\pi R_{c}^{2}=A_{c}=\iint\left|\mu_{12}(\Delta x, \Delta y)\right|^{2} d \Delta x d \Delta y$. Therefore, a Gaussian profile $\left|\mu_{12}\right|$ would be $\left|\mu_{12}(\Delta x, \Delta y)\right|=\exp \left[-\left(\Delta x^{2}\right.\right.$ $\left.\left.+\Delta y^{2}\right) / 2 R_{c}^{2}\right]$.

[27] As reported in Ref. [20], the peak-to-peak divergence angle is $\sim 4.6 \mathrm{mrad} ; 7 \mathrm{mrad}$ contains most of the laser energy. 